\title{
Activating NOTCH3 Mutation
}

National Cancer Institute

\section{Source}

National Cancer Institute. Activating NOTCH3 Mutation. NCI Thesaurus. Code C158058.

A change in the nucleotide sequence of the NOTCH3 gene that that results in constitutive activation of both neurogenic locus notch homolog protein 3 and its downstream signaling pathways. 